\title{
Reliability of controlled linear systems under Gaussian and non-Gaussian loads
}

\author{
Alin Radu ${ }^{1}$ (D) Irina F. Lazar ${ }^{1}$ \\ Received: 26 January 2019 / Revised: 3 April 2019 / Accepted: 9 July 2019 / Published online: 16 July 2019 \\ (c) The Author(s) 2019
}

\begin{abstract}
Civil and mechanical engineering systems are often subjected to vibrations which could alter their behaviour or even lead to their damage or failure. Generated either by man-made processes, such as traffic or equipment, or by natural processes, such as seismic or ocean waves, vibrations may be represented by stochastic processes defined over certain ranges of frequencies. Vibration-control systems have been developed to reduce the undesired response of systems subjected to random vibrations. The aim of the current paper is to compare comprehensively the performance of three popular passive vibrationsuppression devices installed in linear single-degree-of-freedom (SDOF) systems subjected to Gaussian and non-Gaussian random vibrations with general frequency content, characterised by a power-spectral density function. The vibration-control systems analysed in this study are the viscous dampers (VD), the tuned-mass dampers (TMD) and the tuned-inerter dampers (TID), and their performance is assessed in terms of reliability metrics, such as the probability of failure and the first passage time. The twofold goal of this study is reached through (1) the development of explicit analytical relations of the controlledsystems' responses to the Gaussian input; and (2) Monte Carlo simulation estimates of the reliability metrics for the systems subjected to the non-Gaussian loads.
\end{abstract}

Keywords Vibration control $\cdot$ Non-Gaussian vibrations $\cdot$ Random-vibration theory $\cdot$ Reliability analysis $\cdot$ Mean crossing-rates

\section{Introduction}

Mitigation of unwanted vibrations represents an important research topic for many engineering disciplines. This reduction of vibrations is commonly achieved via vibrationisolation systems, i.e. by controlling the supporting structure [1]; or via vibration-suppression systems, i.e. controlling the structure itself by means of supplemental damping $[2,3]$ or other techniques $[4,5]$. This problem can be very challenging given the complexity of engineering systems and the random character of vibrations. The aim of this paper is to provide a comprehensive overview on the performance of vibration-suppression systems, which is achieved through the analysis of the control systems' performance under (1) Gaussian loads, by providing explicit analytical responserelations; and (2) non-Gaussian loads by using estimates of response statistics using Monte Carlo simulations. Unlike

Alin Radu

alin.radu@bristol.ac.uk

1 School of Civil, Aerospace and Mechanical Engineering, University of Bristol, Bristol, UK other similar studies, the both types of loads have a general characterisation of the frequency content given by a powerspectral density function.

Three vibration-suppression systems are analysed, that is, the viscous damper (VD), the tuned-mass damper (TMD) and the tuned-inerter damper (TID). Following is a brief presentation of these systems with a non-exhaustive set of selected references. VDs were introduced in the 70's [6] and are installed to increase the limited inherent structural damping of civil engineering structures $[7,8]$. TMDs were proposed by Frahm as early as 1909 [9] and consist of an added mass, mounted in series with a spring and a damper. Several analytical and numerical tuning methods have been derived since, starting with Den Hartog [10]. While VDs and TMDs have been successful in many engineering applications and installed in several buildings around the world, inerter-based systems, such as the TIDwhere the TMD mass is replaced with an inerter-rely on a newly-introduced device developed by Smith [11]. Since its invention, the inerter has been applied successfully in the automotive industry [12] and in the development of train suspensions $[13,14]$. Recent studies have looked 
into the use of inerters for civil-engineering applications [15-18].

Previous studies regarding the performance of vibration suppressed systems have been carried out before for VDs [2,19], TMDs [20,21], TIDs [4,22], or other control systems such as liquid column dampers [23], vibration barriers [1], or viscoelastic-mass dampers [24], but they generally refer to very specific loading patterns. For example, some studies quantify the performance of the control systems by considering only harmonic or deterministic ground-motion records [4,24-26], while others attempt a probabilistic analysis by using Gaussian white noise [27,28]. Some studies consider more specific frequency patterns, by using Gaussian vibrations with Kanai-Tajimi frequency spectra [29-31], or as in the case of [32], where the TMD is designed to respond to the frequency bandwidth of walking crowds. As for reliability studies on control systems, first- and second- order reliability methods (FORM/SORM) have been used in [27] and a more theoretical approach for the reliability of control theory of random vibrations is shown in [33,34], in which dynamic programming and the stochastic averaging method [35] are used. An analytical solution using crossing theory [36] (Chap. 7.3) for structural control applications is presented in [37], with an application for the tuned mass-damper-inerter in [31].

Similar to some previous studies aforementioned, this paper analyses the response of vibration-controlled linear systems subjected to Gaussian vibrations, but it compares the performances of the VDs, TMDs and TIDs using explicit frequency-response relations for random vibration with general frequency contents. The analytical expressions of the reliability metrics are an essential instrument in designing these systems for random excitation, in order to avoid the expensive Monte Carlo simulations. Explicit analytical relations for reliability metrics have been developed for VD, TMD and TID systems under the assumption of stationary Gaussian input, using elements of random-vibration theory. Analytical solutions are backed by Monte Carlo results, which are further extended for the case of the non-Gaussian vibrations, a relevant distinction between the current research and previous similar studies. The VD-, TMD- and TID-controlled systems' performances are also analysed in the context of the more general non-Gaussian vibrations, with narrow- and broad-band frequency contents. Performing analyses beyond the Gaussian assumption is essential because realistic excitations do not follow it. The non-Gaussian character of loads can be simply shown by calculating the kurtosis coefficient, which for realistic vibration-inducing loads is different than 3 , the value characteristic for Gaussian processes [38]. For example, the kurtosis coefficient is higher than 4 for wind pressure on low-rise structures tested in wind tunnels [39]; is 6.2 for coastal-wave elevations measured in Duck, North Carolina [40]; has an average value of 14.4 for seismic ground-acceleration for earthquakes recorded on rock sites [41]; is only 2.1 for the roughness of a road in South Dakota [38]; and measures 6.2 for the unevenness of a railway track in India [38].

This paper is structured in two main parts: (1) the system and input definitions, that is, the description of the host structure and the vibration-control devices, and the characterisation of the Gaussian and non-Gaussian stochastic processes describing the random vibrations to which the host structure is subjected; and (2) the characterisation of the reliability of the host and vibration-control systems, using random-vibration theory and Monte Carlo simulations, for the Gaussian and non-Gaussian vibrations, respectively. The first part describes the governing equations of motion for the uncontrolled and controlled dynamic systems subjected to random vibrations. For a fair comparison of the controlled systems' performances, the VDs, TMDs and TIDs are calibrated such that the maximum relative displacements of the controlled structures are similar over the entire frequency spectrum. The second part of the paper defines reliability metrics derived using the crossing theory, metrics used for the comparison of the three types of vibration-control systems analysed. Analytical relations for the reliability metrics are developed for the systems subjected to Gaussian input, supported by numerical simulations, used further on for the analyses under non-Gaussian input. Finally, an overall comparison of the increase in the reliability of the controlled structures using all types of controllers, with respect to the uncontrolled host structures, is shown using exceedance probabilities and first-passage times of the critical response.

\section{Systems and input definition}

The goal of the paper is to provide a comprehensive view of the effects of vibration-suppression devices on the performance of dynamic systems subjected to random vibrations. The performance is quantified in terms of its reliability by using elements of random-vibration theory. The problem solved in this paper consists of three main elements, discussed separately in this section: the host dynamic system, the vibration-suppression systems, and the random-vibration input. The host dynamic system is represented by a viscouslydamped linear single-degree-of-freedom (SDOF) system; the vibration-suppression systems analysed are the viscous damper (VD), the tuned mass-damper (TMD) and the tuned inerter-damper (TID); and the random input is represented by stochastic Gaussian and non-Gaussian processes with various frequency contents.

\subsection{Host dynamic system}

The viscously-damped linear SDOF dynamic system subjected to random vibrations is used to analyse the effects of 
(a)

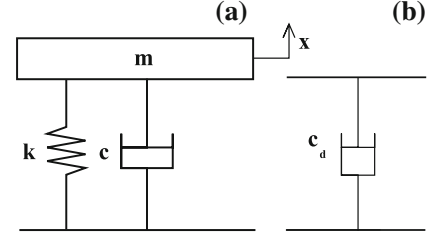

(b)

(c)

(d)

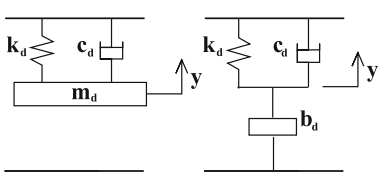

Fig. 1 a Host structure: $v_{0}=\sqrt{k / m}, \zeta_{0}=c /\left(2 \nu_{0} m\right) ; \mathbf{b}$ VD: $\mu=c_{d} / c$; c TMD: $\mu=m_{d} / m, v_{T M D}=\sqrt{k_{d} / m_{d}}, \zeta_{T M D}=c_{d} /\left(2 \nu_{T M D} m_{d}\right)$ and d TID: $\mu=b_{d} / m, v_{T I D}=\sqrt{k_{d} / b_{d}}, \zeta_{T I D}=c_{d} /\left(2 v_{T I D} b_{d}\right)$

vibration-control systems on its performance. Its response is characterized by the following ordinary differential equation

$\ddot{X}(t)+2 \zeta_{0} v_{0} \dot{X}(t)+v_{0}^{2} X(t)=A(t)$

with $t \geq 0$, where $X(t)$ denotes the displacement of the linear SDOF system relative to the ground subjected to a stochastic process $A(t)$. Zero initial conditions, $X(0)=0$ and $\dot{X}(0)=0$, are assumed. Coefficients $\nu_{0}$ and $\zeta_{0}$ are known as the fundamental frequency and damping ratio of the linear SDOF, respectively. Numerical values for the system parameters used for the examples in the following sections are $\nu_{0}=4 \pi \mathrm{rad} / \mathrm{s}$ and $\zeta_{0}=2 \%$.

\subsection{Vibration-suppression systems}

Three types of control systems are used to analyse their vibration-suppression effects on the response of the host structure subjected to Gaussian and non-Gaussian random vibrations. The SDOF host structure and the control systems are shown in Fig. 1. The control systems analysed are modelled inside the host structure, either connected between its mass and the ground (e.g. VD, TID), or connected to the mass only (e.g. TMD), and are used independently to control the vibration in the host structure.

Equation 1 is modified to account for the presence of each control system, as detailed in the following subsections. It should be noted that an SDOF host structure embodying a TMD or a TID becomes a two-degrees-of-freedom system. Note that the rest of the equations of motion for the controlled SDOF system are also written in coordinates relative to the ground.

\subsubsection{VD}

For VDs [2,19], the addition of the damper leads to a direct increase in the structure's damping, with no impact on the natural frequency of the host structure.

$\ddot{X}(t)+2 \zeta_{0}(1+\mu) v_{0} \dot{X}(t)+v_{0}^{2} X(t)=A(t)$

where $\mu=c_{d} / c$ is the ratio between the VD-added damping, $c_{d}$, and the structural damping, $c$. The response $X(t)$ is the displacement of the VD-controlled SDOF linear system relative to the ground.

\subsubsection{TMD}

As noted above, for TMDs the structure becomes more complex, its dynamics being described by the following system of equations

$$
\begin{aligned}
& \ddot{X}(t)+2 \zeta_{0} v_{0} \dot{X}(t)+v_{0}^{2} X(t)+2 \zeta_{T M D} v_{T M D} \mu(\dot{X}(t) \\
& -\dot{Y}(t))+v_{T M D}^{2} \mu(X(t)-Y(t))=A(t) \\
& \ddot{Y}(t)+2 \zeta_{T M D} v_{T M D}(\dot{Y}(t)-\dot{X}(t))+v_{T M D}^{2}(Y(t) \\
& \quad-X(t))=A(t)
\end{aligned}
$$

where $Y(t)$ denotes the displacement response of the TMD system relative to the ground. Zero initial conditions, $Y(0)=$ 0 and $\dot{Y}(0)=0$, are assumed. Coefficients $\nu_{T M D}$ and $\zeta_{T M D}$ are known as the fundamental frequency and damping ratio of the TMD and $\mu$ is the mass ratio between the TMD and the host structure [10]. Note that the respective responses $X(t)$ and $Y(t)$ are coupled and they represent the displacements of the TMD-controlled SDOF linear and the TMD systems, relative to the ground [42].

\subsubsection{TID}

The TID-controlled system response is described by the following set of equations:

$$
\begin{aligned}
& \ddot{X}(t)+2 \zeta_{0} v_{0} \dot{X}(t)+v_{0}^{2} X(t)+2 \zeta_{T I D} v_{T I D} \mu(\dot{X}(t) \\
& -\dot{Y}(t))+v_{T I D}^{2} \mu(X(t)-Y(t))=A(t) \\
& \ddot{Y}(t)+2 \zeta_{T I D} v_{T I D}(\dot{Y}(t)-\dot{X}(t))+v_{T I D}^{2}(Y(t) \\
& \quad-X(t))=0
\end{aligned}
$$

where $Y(t)$ denotes the displacement response of the TID system. Zero initial conditions, $Y(0)=0$ and $\dot{Y}(0)=0$, are assumed. Coefficients $\nu_{T I D}$ and $\zeta_{T I D}$ are known as the fundamental frequency and damping ratio of the TID and $\mu$ is the inertance-to-mass ratio between the TID and the host structure $[4,22]$. Similar to the equations of motion of the TMD, $X(t)$ and $Y(t)$ are coupled and they represent the relative displacements to the ground of the TID-controlled SDOF linear and the TID systems.

The TID is a passive vibration-suppression system, such as the VD or the TMD, that combines the advantages of both the VD and the TMD. Similar to the TMD, the TID introduces an additional degree of freedom to the host structure, with additional damping and stiffness. Unlike the TMD, the TID has its physical mass replaced by the apparent mass produced by the inerter. Similar to the VD, the TID is connected to two terminals of the structure. Thus, the TID is able to reduce 


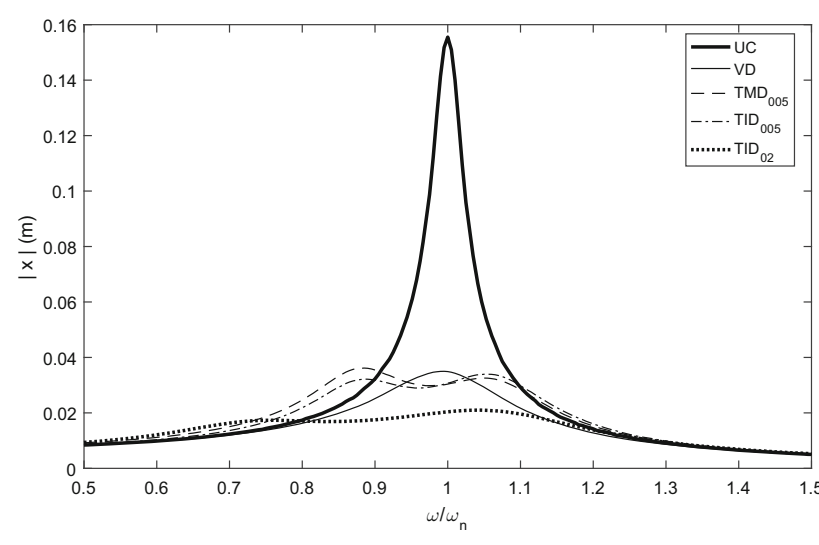

Fig. 2 Frequency response for harmonic excitation with forcing frequency $v$

the system's response at the main frequency, similar to the TMD, but also the response at the other secondary frequencies, similar to the VD. A detailed description of the TID and the equtions of motion of TID-controlled systems are provided in [4], and the similarities between the TID and the TMD in particular can be seen by comparing the respective equations of motion, i.e. Eqs. (4) and (6), respectively.

\subsubsection{Control-systems optimisation}

The goal of the optimisation is obtaining minimum relative displacement of the host structure across the entire frequency range, as shown in Fig. 2. The TMD system is tuned based on Den Hartog's guidelines for damped vibration absorbers, subjected to harmonic base excitation [10]. Den Hartog's analytical optimisation assumes that the host structure is undamped. As mentioned in the previous section, the structure proposed in this paper is lightly damped, having a damping ratio of $\zeta_{0}=2 \%$. The errors introduced by low damping are negligible in practice. Hence, the TMD optimal frequency and damping ratios are given by

$v_{T M D}=\frac{\sqrt{1+\frac{\mu}{2}}}{\mu+1} v_{0}$

$\zeta_{T M D}=\sqrt{\frac{3 \mu}{8(\mu+1)\left(1+\frac{\mu}{2}\right)}}$

A similar analytical optimisation was developed for TIDcontrolled undamped host structures, subjected to base excitation [16]. In the TID case, the optimisation is less sensitive to the structural damping $\zeta_{0}$ of the host structure compared to the TMD. The TID optimal frequency and damping ratios are given by
Table 1 Controlled- and uncontrolled-system parameters

\begin{tabular}{llll}
\hline System & $\nu_{0}[\mathrm{rad} / \mathrm{s}]$ & $\zeta_{0}$ & $\mu$ \\
\hline Uncontrolled $(U C)$ & $4 \pi$ & 0.02 & - \\
Controlled by $V D$ & $4 \pi$ & 0.02 & 3.50 \\
Controlled by $T$ M D $D_{5 \%}$ & $4 \pi$ & 0.02 & 0.05 \\
Controlled by $T$ I $D_{5 \%}$ & $4 \pi$ & 0.02 & 0.05 \\
Controlled by $T$ I I $D_{20 \%}$ & $4 \pi$ & 0.02 & 0.20 \\
\hline
\end{tabular}

$v_{T I D}=\frac{1}{1+\mu} v_{0}$

$\zeta_{T I D}=\sqrt{\frac{3 \mu}{8(1+\mu)}}$

For consistency, it is assumed that the TID and TMD have equal inertance-to-mass and mass ratios, respectively, namely $\mu=5 \%$. Given the inerter's capability of generating amplified mass ratios of up to 200 times larger than its physycal mass [11,43], we consider one additional TID with inertance-to-mass ratio $\mu=20 \%$, which is equivalent to only four times the physical mass of the TMD with $\mu=5 \%$. With an amplified mass ratio, the force generated by the larger TID can bring significant performance improvements over the traditional TMD. The VD is tuned such that the maximum relative displacement at the host structure fundamental frequency matches that of the TID and TMD-controlled structures, when the inertance-to-mass and mass ratios are set to $\mu=5 \%$. Following this consistent calibration rule for the VD, the damping-coefficient ratio that defines the VD results to be $\mu=3.5$. It can be seen from the calibration process described herein, that all three vibrationsuppression systems proposed are essentially defined by one single parameter $\mu$, having different meanings for each system: (1) mass ratio for the TMD; (2) inertance-to-mass ratio for the TID; and (3) damping-coefficient ratio for the VD. A summary of all the parameters for each uncontrolled and controlled structure is shown in Table 1.

\subsection{Random-Vibration input}

The uncontrolled and controlled host structures are subjected to random vibrations, characterized by different frequency contents and Gaussian and non-Gaussian marginal distributions. The random vibrations are defined by a translation stochastic process of the type $A(t)=f[G(t)], t \geq$ 0 , defined in units of acceleration, where $G(t)$ is a stationary zero-mean, unit-variance, Gaussian process with standard Gaussian probability density function $\phi(x)=$ $(2 \pi)^{-0.5} \exp \left\{-0.5 x^{2}\right\}$ and frequency content defined by the one-sided power-spectral density function $g(v), v \geq 0$. Note 


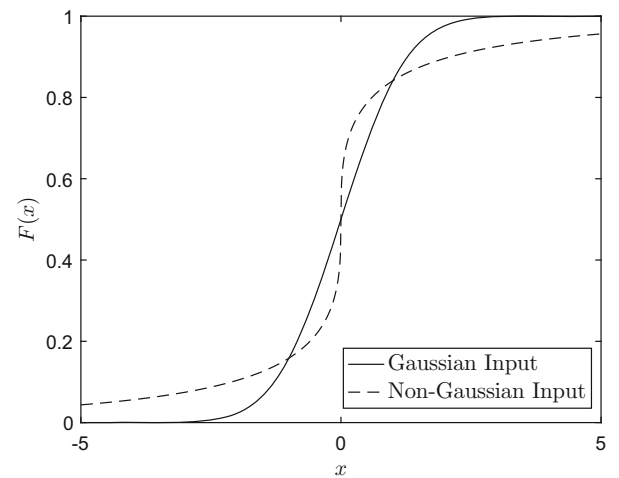

Fig. 3 Marginal distributions of $A(t)$ for $f(x)=x$ (continuous line), and $f(x)=x^{3}$ (dashed line)

that $A(t)$ covers a large class of Gaussian (for $f(x)=x$ ) and non-Gaussian processes (e.g. log-Normal for $f(x)=$ $\exp \{x\}$ ). For our problem we use a standard Gaussian process and a non-Gaussian process defined by the function $f(x)=x^{3}$, to preserve the zero-symmetry of the process. The non-Gaussian process's marginal distribution $F(x)$ has a functional form such that $F^{-1} \circ \Phi(x)=x^{3}$. The marginal distributions for both types of processes are shown in Fig. 3, and it can be noticed that the non-Gaussian defined by $f(x)=x^{3}$ is indeed symmetrical around zero and has considerably larger tails than the Gaussian process. The non-Gaussian distribution chosen has heavier tails than the Gaussian process in this case, as suggested by the data presented in the Introduction section.

For the one-sided spectral-density function we use a function of constant intensity $g_{0}$ defined on a limited frequency bandwidth $\left[v_{a}, v_{b}\right], g(v)=g_{0} \mathbb{1}_{v \in\left[v_{a}, v_{b}\right]}$, where $\mathbb{1}_{v \in\left[v_{a}, v_{b}\right]}$ is called the indicator function and is equal to 1 if $v \in\left[v_{a}, v_{b}\right]$ and 0 , otherwise. Unit variance is assumed for the process, which implies that $g_{0}=\left(v_{b}-v_{a}\right)^{-1}$. Two frequency contents are considered for $A(t)$, i.e. a narrow-band pulse (NBP) frequency with $v_{a}=3 \pi \mathrm{rad} / \mathrm{s}$ and $v_{b}=5 \pi \mathrm{rad} / \mathrm{s}$ defined in the vicinity of the natural frequency of the system, $v_{0}$; and a wide-band frequency with $v_{a}=0$ and $v_{b}=10 \pi \mathrm{rad} / \mathrm{s}$. For the second case, in which $v_{a}=0$, the process $A(t)$ is also known as band-limited white noise (BLWN). The graphical representation of the power-spectral density functions for the two types of processes is shown in Fig. 4a. Any frequency content, whether it is white or "coloured" noise can be used to replace function $g(v)$. The examples chosen in this paper are two general processes, whose frequency ranges can be found in real processes, such as the frequency of people walking for the NBP [44] or of earthquakes for the BLWN [41].

A significant difference between the NBP and the BLWN is seen in their correlation functions. The correlation function is defined as the Fourier transform of the power-spectral density function and can be calculated explicitly for the two cases presented here. Thus, the correlation function for the NBP is
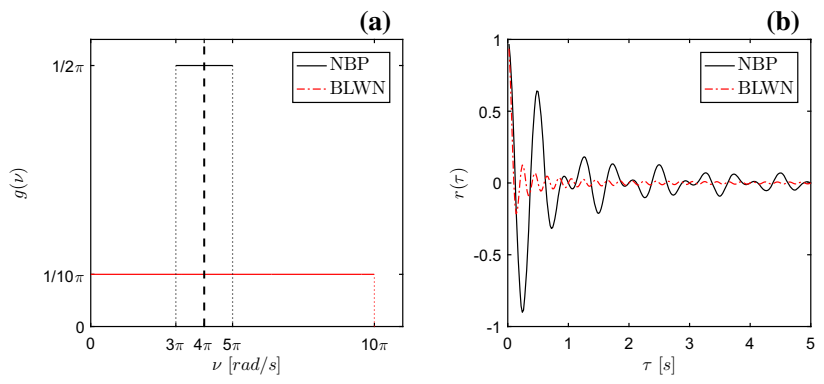

Fig. 4 a Power-spectral density functions $g(v)$; b Correlation functions $r(\tau)$ for the narrow-band and wide-band processes $A(t)$, respectively
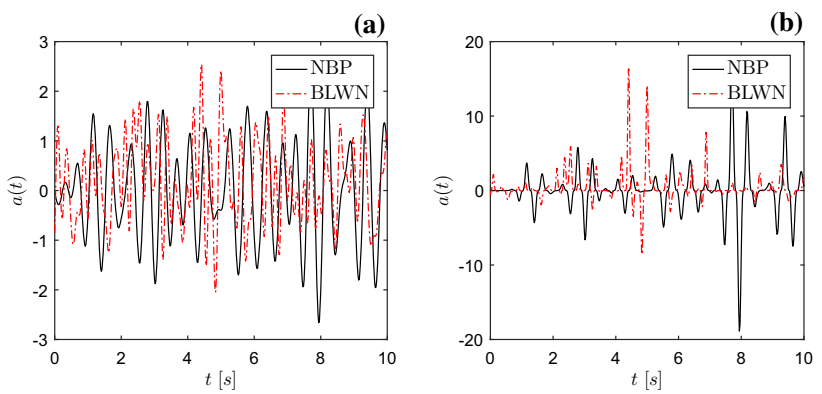

Fig. 5 Samples of $A(t)$ for a the Gaussian process with $f(x)=x$ (left), and $\mathbf{b}$ the non-Gaussian process with $f(x)=x^{3}$

$r(\tau)=2 /\left[\left(v_{b}-v_{a}\right) \tau\right] \sin \left[0.5\left(v_{b}-v_{a}\right) \tau\right] \cos \left[0.5\left(v_{a}+v_{b}\right) \tau\right]$ and for the BLWN is $r(\tau)=1 /\left(v_{b} \tau\right) \sin \left(v_{b} \tau\right)$. Figure $4 \mathrm{~b}$ shows the correlation of the two types of processes for the first $6 \mathrm{~s}$. It can be noticed that the input motion is correlated for the first $1-1.5 \mathrm{~s}$ in the case of the NBP, while it is practically uncorrelated in the case of the BLWN. Figure $5 \mathrm{a}, \mathrm{b}$ show $10 \mathrm{~s}$ samples of the four types of inputs defined, i.e. Gaussian and non-Gaussian, NBP and BLWN processes, respectively. Note that the non-Gaussian samples exhibit high peaks, which is consistent with the distribution functions in Fig. 3. The length of the input samples can be extended to any duration, but given the correlation function in Fig. $4 \mathrm{~b}$ and computational convenience, it was limited to $10 \mathrm{~s}$.

Samples of the process $A(t)$ can be obtained via Monte Carlo simulations using the spectral-representation theorem [45] (Chap. 3.9). The procedure consists mainly of representing the process $A(t)$ as a Fourier series with random coefficients [46].

\section{Evaluation of system performance}

Elements of the random-vibration theory $[35,36]$ and crossing theory $[37,47]$ are used to define the system's performance. Analytical relations can be written for the reliability of systems subjected to Gaussian input [31,37], while for non-Gaussian problems approximate methods have been suggested $[35,48]$, even though the only general and reli- 
able method for calculating reliability metrics remains Monte Carlo. This current study develops explicit analytical relations for the frequency response of the structure controlled with VD, TMD and TID, respectively, by using crossing theory, which absolves the need of using Monte Carlo simulations in the case of the Gaussian assumption, and performs a Monte Carlo-based reliability analysis for the systems subjected to non-Gaussian excitation.

The system performance is evaluated with respect to its response displacement, described by the stochastic process $X(t)$, which is the solution of Eq. (1). The reliability of the viscously-damped linear SDOF system is defined by the probability that its response stays within a safe set $\mathcal{D}$, during its lifetime $\tau$, i.e.

$P_{R}(\tau)=\mathbb{P}\{X(t) \in \mathcal{D}, 0 \leq t \leq \tau\}$,

and its complement $P_{F}(\tau)=1-P_{R}(\tau)$ is known as the probability of failure. The reliability of the system in its lifetime $\tau$ in Eq. (11) can be calculated as the joint probability that the system's initial state is in the safe space $\mathcal{D}$, and that the number of $\mathcal{D}$-outcrossings $N_{\mathcal{D}}(\tau)$, i.e. the number of times the system's response $X(t)$ exits the safe set $\mathcal{D}$, is equal to zero, expressed as $P_{R}(\tau)=\mathbb{P}\left\{(X(0) \in \mathcal{D}) \cap\left(N_{\mathcal{D}}(\tau)=0\right)\right\}$.

Further simplifications can be made to Eq. (11) under the assumption that (i) the state of the initial conditions $X(0)$ and the number of $\mathcal{D}$-outcrossings $N_{\mathcal{D}}(\tau)$ are independent events; (ii) the probability of an instantaneous failure is zero, i.e. $\mathbb{P}\{(X(0) \in \mathcal{D})\}=1$; and (iii) $N_{\mathcal{D}}(\tau)$ follows a non-homogeneous Poisson distribution with rate $\eta_{\mathcal{D}}(t)=\frac{d}{d t} \mathbb{E}\left\{N_{\mathcal{D}}(\tau)\right\}$, called the mean crossing-rate at which the process $X(t)$ exits $\mathcal{D}$. Finally, under the final assumption that (iv) the response $X(t)$ is stationary, i.e., $\eta_{\mathcal{D}}(t)=\eta_{\mathcal{D}}=\mathbb{E}\left\{N_{\mathcal{D}}(\tau)\right\} / \tau$, the reliability of the system in Eq. (11) reduces to

$P_{R}(\tau)=\exp \left\{-\eta_{\mathcal{D}} \tau\right\}$

It must be also added that this simplified equation for the reliability holds only for highly reliable systems, i.e. for low values of $\eta_{\mathcal{D}}$. This is not a limitation when one discusses, for example, the reliability of civil-engineering or aerospaceengineering structures with respect to high thresholds $x_{c r}$. However, if this assumption is unreasonable in some cases, $P_{R}(\tau)$ can be calculated using numerical methods, such as Monte Carlo simulations, as shown in the following section.

An alternative metric for the system's reliability is the firstpassage time $T_{\mathcal{D}}$ [49,50], which, under assumption (ii) is a positive random variable accounting for the time until $X(t)$ crosses outside the safe set $\mathcal{D}$. It is shown in [36] (Chap. 7.4) that the probability distribution of $T_{\mathcal{D}}$ can only be obtained in limited cases and therefore its $k$ th order moments are of interest
$\mathbb{E}\left\{T_{\mathcal{D}}^{k}\right\}=k \int_{0}^{\infty} t^{k-1} P_{R}(t) d t$

where $P_{R}(t)$ is defined in Eq. (12). The moments of $T_{\mathcal{D}}$ exist since

$\lim _{t \rightarrow 0} t^{k-1} \exp \left\{-\eta_{\mathcal{D}} t\right\}=0$, and by replacing $P_{R}(t)$ from Eq. (12) in Eq. (13), it can be shown that the moments of the first-passage time have a closed-form solution dependent only on the mean crossing-rate $\eta_{\mathcal{D}}$, i.e., $\mathbb{E}\left\{T_{\mathcal{D}}^{k}\right\}=k ! / \eta_{\mathcal{D}}^{k}$, where "!" denotes the factorial function. Only the mean and the variance of $T_{\mathcal{D}}$ are used in this paper:

$\mu_{T_{\mathcal{D}}}=\frac{1}{\eta_{\mathcal{D}}}, \quad \sigma_{T_{\mathcal{D}}}^{2}=\frac{1}{\eta_{\mathcal{D}}^{2}}$.

It should be noted that the coefficient of variation of the firstpassage time $T_{\mathcal{D}}$ is equal to 1 , which indicates a reasonablyhigh variability around its mean.

\subsection{Reliability metrics}

In order to evaluate the performance of the uncontrolled linear SDOF system in Eq. (1) and its controlled versions in Eqs. (2), (3) and (5), using the reliability metrics defined in Eqs. (12) and the first two moments of $T_{\mathcal{D}}$ in Eq. (14), we need to evaluate the crossing rates $\eta_{\mathcal{D}}(t)$ for these respective systems. Let $\mathcal{D}=\left[-x_{c r}, x_{c r}\right]$ be a safe set for the response process $X(t)$ of the controlled or uncontrolled linear SDOF system. Since we assumed that the input $A(t)$ and, implicitly, $X(t)$ are symmetric about zero, we can state that the $\mathcal{D}$-outcrossing rate of $X(t)$ is $\eta_{\mathcal{D}}=2 \eta_{\mathcal{D}}^{+}$, where $\eta_{\mathcal{D}}^{+}$represents the $x_{c r}$-upcrossings rate of $X(t)$, or the rate at which $X(t)$ would cross the upper level $x_{c r}$ with positive slope. In other words, $\eta_{\mathcal{D}}^{+}$can be calculated as the probability of $X(t)$ crossing the level $x_{c r}$ with positive slope in an infinitesimal interval of time $(t, t+\Delta t)$, i.e.

$\eta_{\mathcal{D}}^{+}=\lim _{\Delta t \rightarrow 0} \frac{1}{\Delta t} \mathbb{P}\left\{X(t) \leq x_{c r} \leq X(t+\Delta t)\right\}$

The upcrossing rate $\eta_{\mathcal{D}(t)}^{+}$can be calculated using the Rice formula [47] (Chap.10) by approximating $X(t+\Delta t)$ by $X(t)+\dot{X}(t) \Delta t$ from the first-order finite difference of $\dot{X}(t)$, provided that the probability distribution function of $[X(t), \dot{X}(t)]$ is known. Numerically, the mean crossing-rate $\eta_{\mathcal{D}}$, for given levels $x_{c r}$ of the safety set $\mathcal{D}$, is calculated by counting the number of $x_{c r}$-upcrossings and $x_{c r}$ downcrossings (the rate at which $X(t)$ crosses the lower level $-x_{c r}$ with negative slope) over the time period $\tau$. Figure 6 a shows the number of $x_{c r}$-upcrossings and $x_{c r}$-downcrossings for a sample of the response for a critical level $x_{c r}=0.05$, over a time window of $10 \mathrm{~s}$. Figure $6 \mathrm{~b}$ shows the crossing rates calculated for 10,000 samples of the response $X(t)$ 

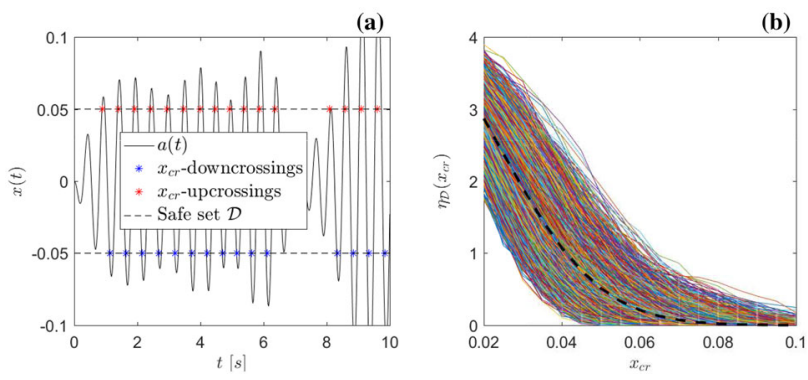

Fig. 6 a $\mathcal{D}$-outcrossings of a sample $x(t)$ of $X(t)$ for $x_{c r}=0.05$, $\mathbf{b}$ crossing rates for 10,000 samples of $X(t)$ and their mean crossing-rate $\eta_{\mathcal{D}}$ in bold, dashed black line

subjected to the non-Gaussian NBP $A(t)$, and the mean crossing-rate of $X(t)$ in black-dashed line.

For the particular case in which $A(t)$ is Gaussian, it can be inferred that $[X(t), \dot{X}(t)]$ is also Gaussian with zero mean and variance $\left[\dot{\sigma}_{X}^{2}(t), \sigma_{X}^{2}(t)\right]$, parameters which are calculated further down in this section. The mean crossing-rate $X(t)$ follows to be

$\eta_{\mathcal{D}}(t)=\frac{\dot{\sigma}_{X}(t)}{\pi \sigma_{X}(t)} \exp \left\{-\frac{x_{c r}^{2}}{2 \sigma_{X}^{2}(t)}\right\}$,

as also shown in [36] (Chap. 7.3). Eq. (16) can be further developed and explicit relations for the mean crossing-rates of the systems controlled by the VD, TMD and TID, respectively, can be calculated under the Gaussian assumption. These relations will be beneficial for calculating reliability metrics in this case, without expensive Monte Carlo simulations. The analytical solution for the response of viscously-damped linear SDOF oscillators described in Eq. (1) is known

$\left[\begin{array}{c}X(t) \\ \dot{X}(t)\end{array}\right]=\int_{0}^{t} \boldsymbol{\Phi}(t-u)\left[\begin{array}{c}0 \\ A(u)\end{array}\right] d u$

where the transfer matrix for the linear oscillator is

$$
\begin{aligned}
& \boldsymbol{\Phi}(t)=e^{-\zeta_{0} v_{0} t} \times \\
& {\left[\begin{array}{cc}
\cos \left(v_{d} t\right)+\frac{\zeta_{0} v_{0}}{v_{d}} \sin \left(v_{d} t\right) & \frac{1}{v_{d}} \sin \left(v_{d} t\right) \\
-\frac{v_{0}^{2}}{v_{d}} \sin \left(v_{d} t\right) & \cos \left(v_{d} t\right)-\frac{\zeta_{0} v_{0}}{v_{d}} \sin \left(v_{d} t\right)
\end{array}\right],}
\end{aligned}
$$

and $v_{d}=v_{0} \sqrt{1-\zeta_{0}^{2}}$ is the damped natural frequency. The variances of $X(t)$ and $\dot{X}(t)$ used in Eq. (16) are calculated directly:

$$
\begin{aligned}
\sigma_{X}^{2}(t)= & \int_{v_{a}}^{v_{b}} \int_{0}^{t} \int_{0}^{t} \phi_{12}(t-u) \phi_{12}(t-v) g(v) \\
& \cos (v(u-v)) d u d v d v
\end{aligned}
$$

Table 2 Transfer function terms for the uncontrolled system (UC), VDs and TMDs

\begin{tabular}{lll}
\hline & UC & VD \\
\hline D & 1 & 1 \\
E & 0 & 0 \\
F & $1-\left(v / \nu_{0}\right)^{2}$ & $1-\left(v / \nu_{0}\right)^{2}$ \\
G & $2 \zeta_{0} v / \nu_{0}$ & $2 \zeta_{0}(1+\mu) v / \nu_{0}$ \\
\hline
\end{tabular}

Table 3 Transfer function terms for TMDs

\begin{tabular}{ll} 
& $\mathrm{TMD}$ \\
\hline $\mathrm{D}$ & $\mu\left(\nu / \nu_{0}\right)^{2}-(1+\mu) \gamma$ \\
$\mathrm{E}$ & $2(1+\mu) \beta \zeta_{0} \nu / \nu_{0}$ \\
$\mathrm{~F}$ & $\mu\left(\nu / \nu_{0}\right)^{4}-\left(\gamma+\mu+\gamma \mu+4 \beta \zeta_{0}^{2}\right)\left(\nu / \nu_{0}\right)^{2}+\gamma$ \\
$\mathrm{G}$ & $2 \zeta_{0}\left((\beta+\mu+\beta \mu)\left(\nu / \nu_{0}\right)^{3}-(\beta+\gamma)\left(\nu / \nu_{0}\right)\right)$ \\
\hline
\end{tabular}

$$
\begin{aligned}
\dot{\sigma}_{X}^{2}(t)= & \int_{v_{a}}^{v_{b}} \int_{0}^{t} \int_{0}^{t} v^{2} \phi_{22}(t-u) \phi_{22}(t-v) g(v) \\
& \cos (v(u-v)) d u d v d v
\end{aligned}
$$

where $\phi_{12}(t)$ and $\phi_{22}(t)$ are the $(1,2)$ and $(2,2)$ elements of the transfer matrix $\boldsymbol{\Phi}(t)$ in Eq. (18), respectively. The triple integrals are difficult to solve, but solutions can be found numerically.

Note that the response process $X(t)$ may not be stationary, although the input $A(t)$ is, as it can be seen also in Fig. 6a. However, under the stated problem, it has been shown that the second moments converge quickly to the stationary solution even for low damping ratios [36]. Calculation of $\sigma_{X}^{2}(t)$ and $\dot{\sigma}_{X}^{2}(t)$ can be simplified further under the stationarity assumption, which leads to

$\sigma_{X}^{2}=\int_{v_{a}}^{v_{b}}|h(v)|^{2} g(v) d v$

$\dot{\sigma}_{X}^{2}=\int_{v_{a}}^{v_{b}} v^{2}|h(v)|^{2} g(v) d v$

where

$|h(v)|=\frac{1}{v_{0}} \sqrt{\frac{D^{2}+E^{2}}{F^{2}+G^{2}}}$

is the modulus of the transfer function between $X(t)$ and $A(t)$, with coefficients in Tables 2, 3 and 4. Coefficients $\gamma=$ $\mu\left(v_{T M D} / v_{0}\right)^{2}$ and $\beta=\mu \zeta_{T M D} v_{T M D} /\left(\zeta_{0} v_{0}\right)$ in Table 3 , and $\gamma=\mu\left(\nu_{T I D} / \nu_{0}\right)^{2}$ and $\beta=\mu \zeta_{T I D} \nu_{T I D} /\left(\zeta_{0} \nu_{0}\right)$ in Table 4 can be calculated simply using the $\mu$ parameter defined by the calibration of each vibration-control system, shown in Table 1. The mean crossing-rates $\eta_{\mathcal{D}}$ of the uncontrolled response of the system in Eq. (1) have been calculated for 
Table 4 Transfer function terms for TIDs

TID

\begin{tabular}{ll}
\hline $\mathrm{D}$ & $\mu\left(\nu / \nu_{0}\right)^{2}-\gamma$ \\
$\mathrm{E}$ & $2(1+\mu) \beta \zeta_{0} \nu / \nu_{0}$ \\
$\mathrm{~F}$ & $\mu\left(\nu / \nu_{0}\right)^{4}-\left(\gamma+\mu+\gamma \mu+4 \beta \zeta_{0}^{2}\right)\left(\nu / \nu_{0}\right)^{2}+\gamma$ \\
$\mathrm{G}$ & $2 \zeta_{0}\left((\beta+\mu+\beta \mu)\left(\nu / \nu_{0}\right)^{3}-(\beta+\gamma)\left(\nu / \nu_{0}\right)\right)$ \\
\hline
\end{tabular}
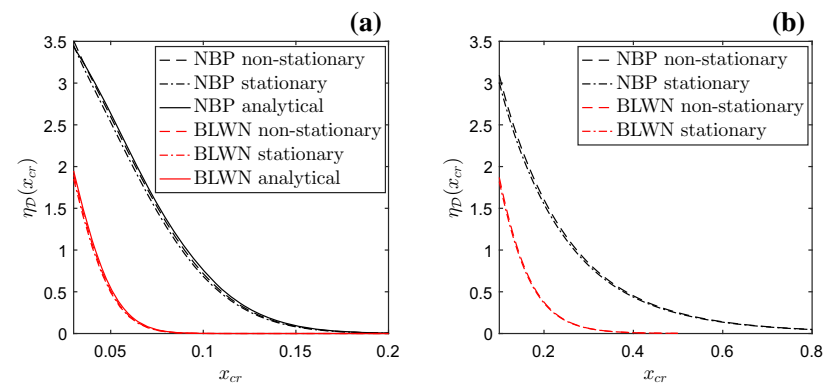

Fig. 7 Mean crossing-rates $\eta_{\mathcal{D}}$ for the uncontrolled response $X(t)$ under the a Gaussian and $\mathbf{b}$ non-Gaussian NBP and BLWN, respectively

both the Gaussian and the non-Gaussian, NBP and the BLWN motions $A(t)$, as defined in Sect. 2.3.

Figure $7 \mathrm{a}, \mathrm{b}$ show the mean crossing-rates $\eta_{\mathcal{D}}$ for the response under Gaussian and non-Gaussian processes, respectively. The analytical values for $\eta_{\mathcal{D}}$ can be calculated using Eq. (16) only for the Gaussian input. Numerical values of the mean crossing-rates have been calculated in two ways, i.e., using the entire sample of the response $X(t), t \geq 0$, and by disregarding the initial few seconds of the transient response for $t \geq t_{s}$. Given the negligible differences between the mean crossing-rates calculated by considering and disregarding the transient part of $X(t)$, the stationarity assumption can be adopted further on. The results in Fig. 7 support the stationarity assumption given the almost identical mean crossing-rates calculated analytically using Eqs. (19) and (20), and numerically via Monte-Carlo simulations described in detail below.

It must be noted that Eqs. (21)-(23) are valid for any power-spectral density function $g(v)$, and not only for the constant-intensity functions used in this paper. However, the choice of the white-noise functions is relevant for engineering applications with low damping ratios $\zeta_{0}$ and relatively smooth power-specral density functions $g(v)$. Under these conditions, $|h(v)|$ is sharply peaked at $v_{0}$ in the case of the uncontrolled host, and it has been shown that the main contribution of the response can be approximated by setting $g(v)=g\left(v_{0}\right)$ to be constant [51].

The same approach can be applied in the case of the controlled structures, by updating $|h(v)|$ accordingly. However, for more complex systems, the number of terms in the expression of the corresponding transfer functions increases significantly. The expressions for coefficients $D, E, F$ and $G$ in Eq. 23 can be found in Tables 2-4.

All the analytical relations developed herein in detail are valid for linear host systems subjected to Gaussian input $A(t)$. As already mentioned previously, the results shown for the non-Gaussian input, as well as the validation of the analytical solutions are done using Monte Carlo simulations. Thus, if $a_{k}(t), k=1, \ldots, N$ are $N$ samples of the process $A(t)$, then $x_{k}(t)$ are the corresponding response samples of (1) the uncontrolled system in Eq. (1); (2) the VD-controlled system in Eq. (2), (3) the TMD-controlled system in Eq. (3), or (4) the TID-controlled system in Eq. (5), calculated by solving each of these equations respectively. Then, similarly to the approach described earlier, the mean upcrossing-rate in Eq. (15) can be approximated using the Monte Carlo simulations as

$\eta_{\mathcal{D}}^{+}=\frac{1}{N} \sum_{k=1}^{N} \frac{1}{\tau} \sum_{i=1}^{n_{t}} \mathbb{1}\left(\left|x_{k}\left(t_{i}\right)\right|>x_{c r}\right)$,

where index $i=1, \ldots, n_{t}$ denotes the discrete times $0 \leq$ $t_{i} \leq \tau$ at which $a(t)$ is sampled, and function $\mathbb{1}$ denotes the counting indicator function. The mean upcrossing-rates $\eta_{\mathcal{D}}^{+}$calculated either by Eq. (15) or by Eq. (24) are used to derive all the reliability metrics described herein. Further on, the expression for the reliability in Eq. (12) is only valid for small $\eta_{\mathcal{D}}^{+}$, but using Monte Carlo simulations, it can be calculated by relaxing this assumption, using the following relation:

$P_{R}(\tau)=\frac{1}{N} \sum_{k=1}^{N} \mathbb{1}\left(\max _{0 \leq t \leq \tau}\left|x_{k}(t)\right| \leq x_{c r}\right)$.

Even though the relations for the Gaussian case have been developed previously, for consistency Eqs. (24) and (25) are used to calculate the results for the comparison between the reliability of the systems subjected to both types of Gaussian and non-Gaussian loads.

\subsection{Reliability analysis of controlled systems}

The goal of this section is to use the reliability metrics defined above to assess the effect of the control systems designed and calibrated in Sect. 2.2 on the performance of the uncontrolled host structure. The mean crossing-rates defined in Eq. (16) can be calculated analytically for the case of stationary Gaussian input with the aid of Eqs. (21)-(23), for both the uncontrolled and controlled systems, or numerically using Monte Carlo simulations for any type of loading. Figures 8 and 9 show the mean crossing-rates for the VD-, TMD- and TID-controlled systems in comparison with the mean crossing-rates for the host structure, for the Gaussian 

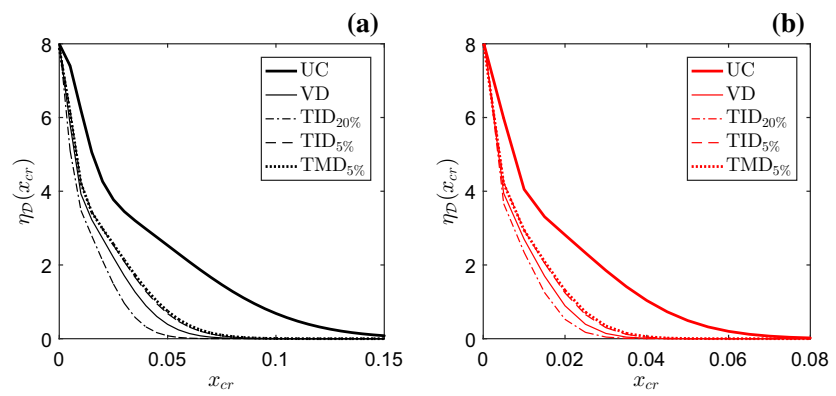

Fig. 8 Mean crossing-rates $\eta_{\mathcal{D}}$ for the controlled response $X(t)$ under the Gaussian a NBP and b BLWN
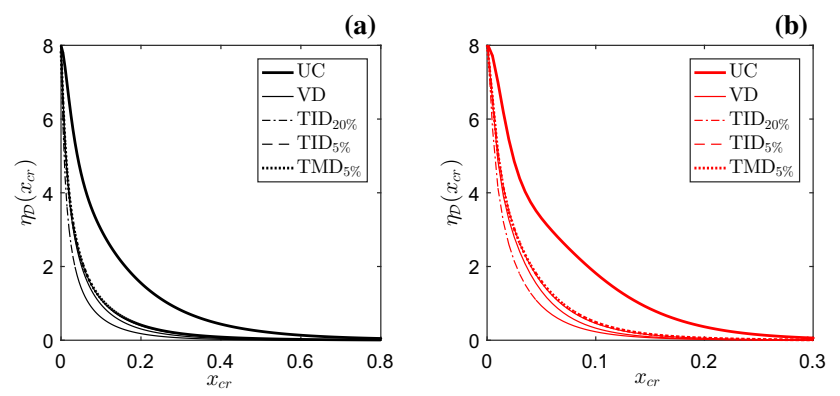

Fig. 9 Mean crossing-rates $\eta_{\mathcal{D}}$ for the controlled response $X(t)$ under the Non-Gaussian a NBP and b BLWN

and non-Gaussian input, respectively. Since the analytical solutions have already been validated previously against the numerical results, all the results in this section, for both types of stochastic processes, are calculated using Monte Carlo simulations by the approach described in Eqs. (24) and (25). In each of the two figures, (a) and (b) panels show the mean crossing-rates of the controlled systems for the BNP and BLWN, respectively. The responses of all systems are higher for the NBP input, since most of the energy of the input is concentrated around the natural frequency of the systems, a fact that can also be seen in Fig. 9. As expected, the mean crossing-rates are considerably higher for the non-Gaussian motion due to its higher peaks, generated by the heavier tails of its distribution, as seen in Fig. 3.

The mean crossing-rates are a measure of the rate at which the response of a system goes above a threshold $x_{c r}$. Both figures are calculated for a time window $\tau=10 \mathrm{~s}$, and show a decrease in the mean crossing-rates of the controlled systems, which translates into an increase of the system performance. The TMD and TID with $\mu=5 \%$ display almost identical behaviour, a fact foreseen by following the tuning methodology, illustrated graphically in Fig. 2. Figure 2 also suggests that the VD would perform better with respect to the TMD and TID with $\mu=5 \%$, due to its lower response outside a small vicinity around the natural frequency $\nu_{0}$. However, this is only reflected in Fig. 8, in the case of the Gaussian input, and less so for the non-Gaussian case. One needs to consider the fact that the control devices have been designed for har-
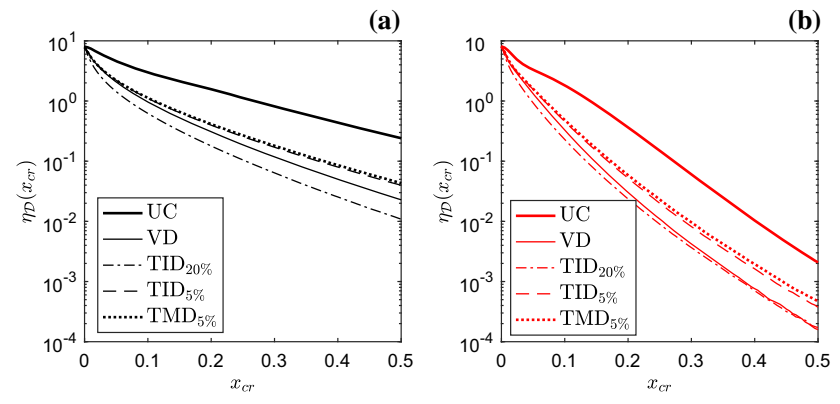

Fig. 10 Mean crossing-rates $\eta_{\mathcal{D}}$ for the controlled response $X(t)$ under non-Gaussian a NBP and b BLWN, in logarithmic scale

monic oscillations, and not specifically for the random input, which is beyond the purpose of the current study. A considerable improvement in the response of the host structure has been achieved using the analytical tuning methodology described in Sect. 2.2.4, and a design targeted towards random inputs would only reinforce the conclusions regarding the performance of the controlled structures. Given the capability of the inerter to have its apparent mass (i.e. its inertance) increased without a significant increase in its physical mass, the TID with $\mu=20 \%$ represents a more reliable option. This is verified by reduced mean crossing-rates achieved by using this device. This characteristic is valid for both the Gaussian and non-Gaussian excitations, proving the TID to be a more reliable and robust control device in comparison with the VD and the TMD which becomes unrealistic for $\mu>10 \%$.

Figure 9a, b show the mean crossing-rates for the nonGaussian NBP and BLWN processes, respectively. Given the amplitudes of the response in the case of the non-Gaussian input, the mean crossing-rates at extreme values of $x_{c r}$ are examined on a logarithmic scale. Figure 10 shows a representation of mean crossing-rates of the systems for the non-Gaussian input, shown in Fig. 9. It can be noticed that a considerable reduction in the mean crossing-rates is achieved with all control systems.

The mean crossing-rates shown in Figs. 8, 9 and 10 were calculated using Monte Carlo simulations and Eq. (24), and give a fair idea of the performance of the maximum response of the controlled systems. However, for specified levels of design $x_{c r}$, probabilities of failure, $P_{F}(\tau)=1-P_{R}(\tau)$, or mean first-passage times $\mathbb{E}\left[T_{\mathcal{D}}\right]$ are more commonly used metrics of reliability in the engineering community. Thus, Figs. 11, 12, 13 and 14 show the probabilities of failure $P_{F}\left(\tau, x_{c r}\right)$ for a range of safe sets represented by $x_{c r}$. These results were obtained by calculating the reliability of each system, using Monte Carlo simulations and the estimate described in Eq. (25).

Figure 11 shows $P_{F}\left(\tau, x_{c r}\right)$ for the host and controlled systems subjected to the Gaussian NBP input. By comparing the four panels of the figure, it can be seen how the plateau 

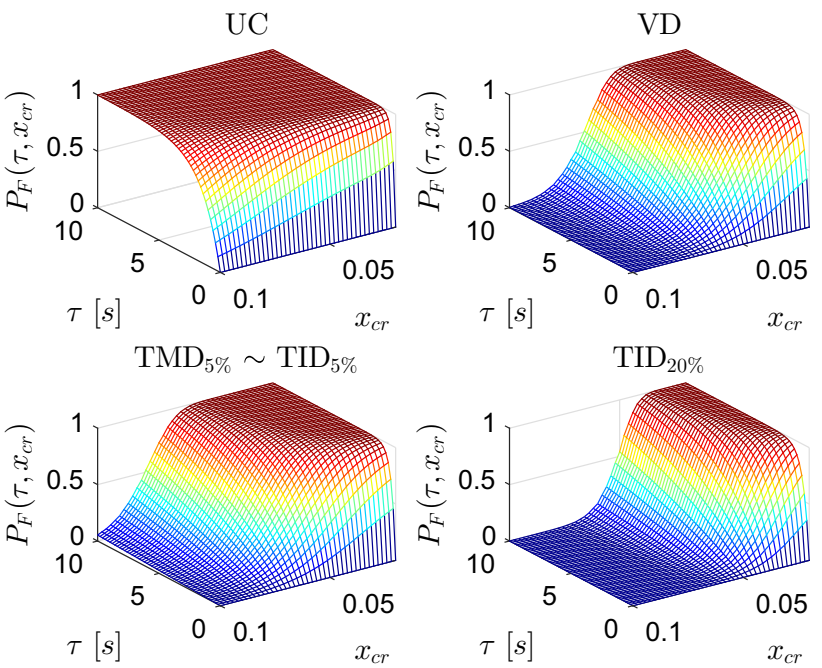

Fig. 11 Probability of failure of controlled systems under Gaussian NBP loading
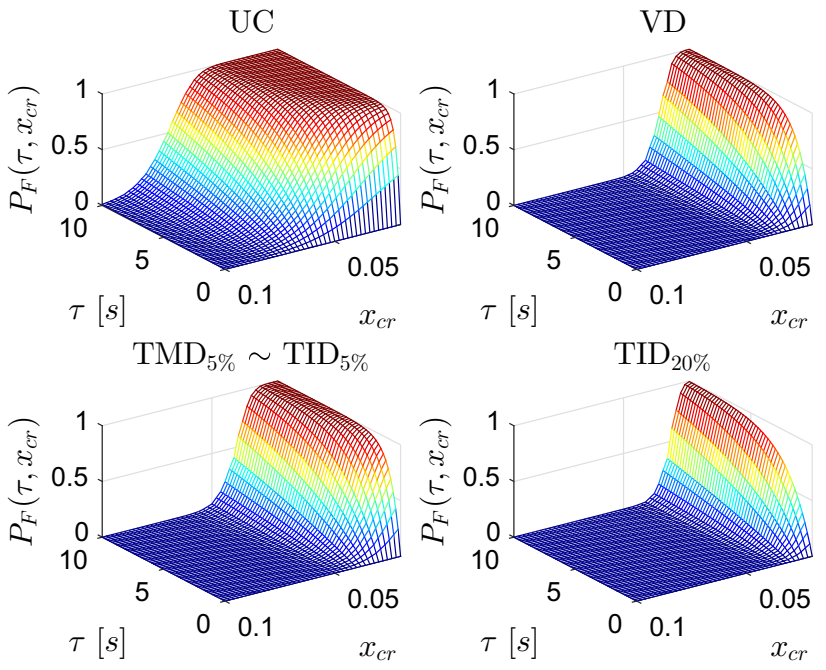

Fig. 12 Probability of failure of controlled systems under Gaussian BLWN loading

region where $P_{F}\left(\tau, x_{c r}\right)=1$ shrinks after the addition of control devices. The trend is consistent with the results shown in Fig. 8a, where the mean crossing-rates suggest that the best performing system is the TID with $\mu=20 \%$, followed by the VD and the TMD and TID with $\mu=5 \%$. The same observations apply to Fig. 12 showing $P_{F}\left(\tau, x_{c r}\right)$ for the host and controlled systems subjected to the Gaussian BLWN input, if analysed in conjunction with Fig. 8(b).

Comparing the failure plateaus in Figs. 11 and 12 confirms that the higher concentration of vibration energy around $v_{0}$ triggers higher structural response. Figure 13 shows the $P_{F}\left(\tau, x_{c r}\right)$ for the systems subjected to the non-Gaussian NBP input. The results correlate with the mean crossingrates in Fig. 9a, where the gap between the performance of the VD versus the TMD and TID with $\mu=5 \%$ is reduced.
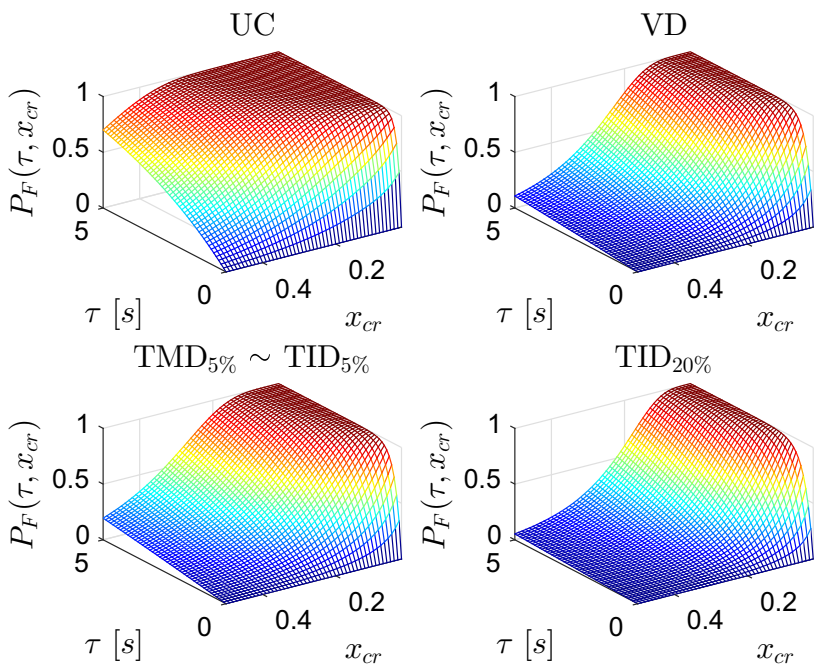

Fig. 13 Probability of failure of controlled systems under NonGaussian NBP loading
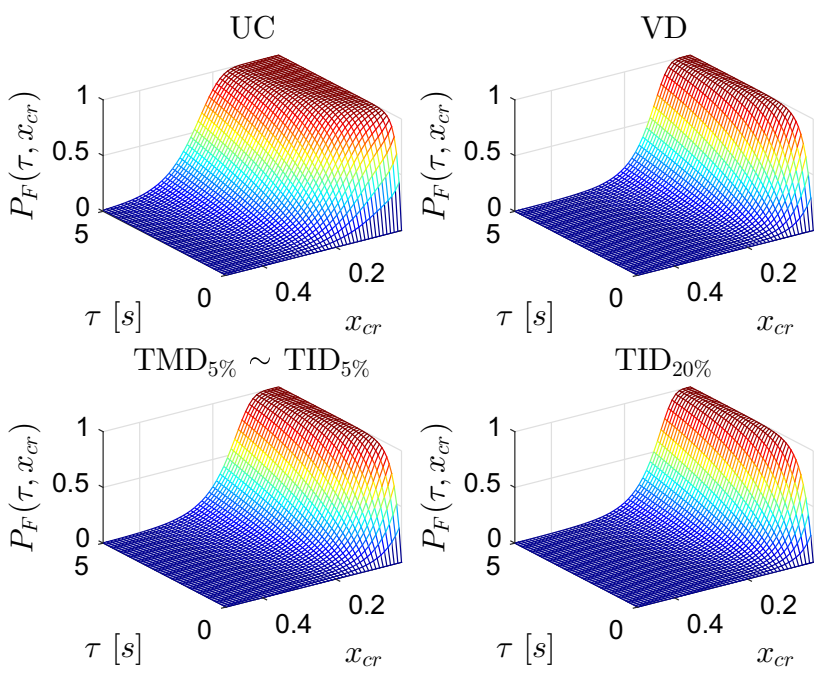

Fig. 14 Probability of failure of controlled systems subjected to nonGaussian BLWN loading

Figure 14 shows $P_{F}\left(\tau, x_{c r}\right)$ for the host and controlled systems subjected to the non-Gaussian BLWN input. Comparing Figs. 13 and 14, a decrease in performance is observed in the case of the BLWN.

Finally, Fig. 15 presents the performance of the vibrationcontrolled systems in terms of the means and standard deviations of the first-passage times on a logarithmic scale. The left and right panels show the mean times for the systems subjected to the Gaussian and non-Gaussian input for both types of motions, i.e. NBP in black and BLWN in red. As noticed before, the control systems increase the reliability of the host structure in all cases, by increasing the crossing times for a specified value of the threshold $x_{c r}$. For example, the mean first-passage time of a threshold $x_{c r}=0.2$ increases by approximately a factor of 5,4 , and 9 times, respectively, when 

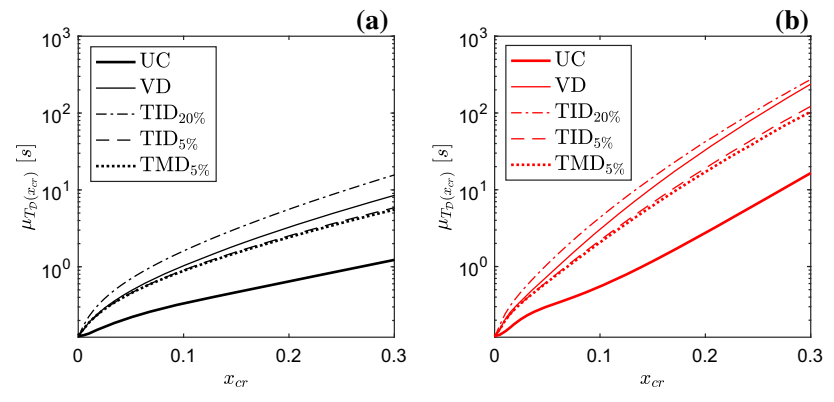

Fig. 15 Mean first-passage time $\mu_{T_{\mathcal{D}}}$ (and standard deviation $\sigma_{T_{\mathcal{D}}}$ ) of the systems subjected to non-Gaussian a NBP and b BLWN input

a VD, a TMD/TID with $\mu=5 \%$, or a TID with $\mu=20 \%$ is installed in the host structure subjected to the non-Gaussian NBP, and by approximately a factor of 12, 7 and 15 times in the case of the non-Gaussian BLWN. All three types of devices, i.e. VD, TMD and TID, perform well, as noted previously, but are less efficient in the case of the non-Gaussian vibrations due to their pulse-like character, with high isolated peaks.

\section{Conclusion}

This paper offered a comparative overview of the gain in reliability of vibration-controlled systems with commonlyused devices, i.e. VD, TMD and TID, for structures subjected to random vibrations. Linear SDOF systems subjected to both Gaussian and non-Gaussian stationary vibrations with various frequency content were used as host structures. Reliability metrics in terms of probability of failure and moments of the first-passage times of the system to exit the displacement safe range, have been provided using crossing theory. Explicit analytical relations were provided for all types of systems subjected to Gaussian input, and have been backed by numerical results obtained by Monte Carlo simulations, extended also for the non-Gaussian case.

It has been shown that a considerable gain in reliability of the host structure can be achieved for all loading patterns (BLP and BLWN Gaussian and non-Gaussian inputs) using vibration-control systems tuned such that they display similar maximum displacement response over the entire frequency range. The study not only allowed for a parallel comparison between different types of vibration-suppression devices, but also showed that they are efficient for a large range of stochastic processes with different frequency contents and different probability laws. Moreover, the TID is more robust and reliable than the VD and TMD.

Acknowledgements The work of Alin Radu reported in this paper has been supported by the Marie Skłodowska-Curie Actions of the European Union's Horizon 2020 Program under the Grant Agreement 704679 - PARTNER. This support is gratefully acknowledged.
Funding AR was funded by the Marie Skłodowska-Curie Actions of the European Union's Horizon 2020 Program under the Grant Agreement No. 704679 - PARTNER.

\section{Compliance with Ethical Standards}

Conflict of Interest The authors declare that they have no conflict of interest.

Open Access This article is distributed under the terms of the Creative Commons Attribution 4.0 International License (http://creativecomm ons.org/licenses/by/4.0/), which permits unrestricted use, distribution, and reproduction in any medium, provided you give appropriate credit to the original author(s) and the source, provide a link to the Creative Commons license, and indicate if changes were made.

\section{References}

1. Cacciola P, Tombari A (2015) Vibrating barrier: a novel device for the passive control of structures under ground motion. Proc R Soc Lond A Math Phys Eng Sci 471(2179):1-22. https://doi.org/10. 1098/rspa.2015.0075

2. Güneyisi EM, Altay G (2008) Seismic fragility assessment of effectiveness of viscous dampers in $\mathrm{r} / \mathrm{c}$ buildings under scenario earthquakes. Struct Saf 30(5):461-480

3. Prodan O, Ladar I, Alexa P (2010) Seismic performances of steel structures with supplementar damping. Numerical studies. In: Proceedings of the 14th European conference on earthquake engineering. Ohrid, Republic of Macedonia, pp. 476-482

4. Lazar IF, Neild S, Wagg D (2014) Using an inerter-based device for structural vibration suppression. Earthq Eng Struct Dyn 43(8):1129-1147

5. Papadimitriou C, Katafygiotis LS, Au S-K (1997) Effects of structural uncertainties on tmd design: a reliability-based approach. J Struct Control 4(1):65-88

6. Pervyshi VG, Naimov PI (1970) Performance of a viscous-friction torsional-vibration damper. Russ Eng J URSS 50(11):19

7. Constantinou MC, Symans MD (1992) Experimental and analytical investigation of seismic response of structures with supplemental fluid viscous dampers. Report No. NCEER- 92-0032, National Center for Earthquake Engineering Research, Buffalo, NY, USA

8. Constantinou MC, Symans MD (1970) Seismic response of structures with supplemental damping. Struct Des Tall Build 2:77-92

9. Frahm H (1909) Device for damping vibrations of bodies. Patent Number US989958 A

10. DenHartog JP (1947) Mechanical vibrations. McGraw-Hill, New York

11. Smith MC (2002) Synthesis of mechanical networks the inerter. IEEE Trans Autom Control 47:1648-1662

12. Smith MC, Wang F-C (2004) Performance benefits in passive vehicle suspensions employing inerters. Veh Syst Dyn 42(4):235-257

13. Jiang JZ, Matamoros-Sanchez AZ, Zolotas A, Goodall RM, Smith MC (2015) Passive suspensions for ride quality improvement of two-axle railway vehicles. Proc Inst Mech Eng Part F J Rail Rapid Transit 229(3):315-329

14. Wang F-C, Hsieh M-R, Chen H-J (2012) Stability and performance analysis of a full-train system with inerters. Veh Syst Dyn 50(4):545-571

15. Takewaki I, Murakami S, Yoshitomi S, Masaaki T (2012) Fundamental mechanism of earthquake response reduction in building structures with inertial dampers. Struct Control Health Monit 19:590-608 
16. Gonzalez-Buelga A, Lazar IF, Jiang JZ, Neild SA, Inman DJ (2017) Assessing the effect of nonlinearities on the performance of a tuned inerter damper. Struct Control Health Monit 24(3):1-17. https:// doi.org/10.1002/stc. 1879

17. Ikago K, Saito K, Inoue N (2012) Seismic control of single-degreeof-freedom structure using tuned viscous mass damper. Earthq Eng Struct Dyn 41(3):453-474

18. Sugimura Y, Goto W, Tanizawa H, Saito K, Ninomiya T, Nagasaku T, Saito K (2012) Response control effect of steel building structure using tuned viscous mass damper. Proceedings of the 15 th world conference on earthquake engineering

19. Yang G, Spencer B, Carlson J, Sain M (2002) Large-scale mr fluid dampers: modeling and dynamic performance considerations. Eng Struct 24(3):309-323

20. Li C (2000) Performance of multiple tuned mass dampers for attenuating undesirable oscillations of structures under the ground acceleration. Earthq Eng Struct Dyn 29(9):1405-1421

21. Lin C-C, Ueng J-M, Huang T-C (2000) Seismic response reduction of irregular buildings using passive tuned mass dampers. Eng Struct 22(5):513-524

22. Marian L, Giaralis A (2014) Optimal design of a novel tuned mass-damper-inerter (tmdi) passive vibration control configuration for stochastically support-excited structural systems. Probab Eng Mech 38:156-164

23. Taflanidis AA, Beck JL, Angelides DC (2007) Robust reliabilitybased design of liquid column mass dampers under earthquake excitation using an analytical reliability approximation. Eng Struct 29(12):3525-3537

24. Muresan CI, Dulf EH, Prodan O (2014) A fractional order controller for seismic mitigation of structures equipped with viscoelastic mass dampers. J Vib Control 22(8):1980-1992

25. Lazar IF, Neild S, Wagg D (2016) Vibration suppression of cables using tuned inerter dampers. Eng Struct 122:62-71

26. Pietrosanti D, De Angelis M, Basili M (2017) Optimal design and performance evaluation of systems with tuned mass damper inerter (tmdi). Earthq Eng Struct Dyn 46(8):1367-1388

27. Spencer B, Sain M, Won C-H, Kaspari D, Sain P (1994) Reliability-based measures of structural control robustness. Struct Saf 15(1):111-129 Special Issue on Reliability on Special Structural Systems

28. Marano GC, Greco R (2011) Optimization criteria for tuned mass dampers for structural vibration control under stochastic excitation. J Vib Control 17(5):679-688

29. Chakraborty S, Roy BK (2011) Reliability based optimum design of tuned mass damper in seismic vibration control of structures with bounded uncertain parameters. Probab Eng Mech 26(2):215-221

30. Yu H, Gillot F, Ichchou M (2013) Reliability based robust design optimization for tuned mass damper in passive vibration control of deterministic/uncertain structures. J Sound Vib 332(9):2222-2238

31. Giaralis A, Taflanidis AA (2015) Reliability-based design of tuned mass-damper-inerter (tmdi) equipped multi-storey frame buildings under seismic excitation. In: Proceedings of the 12th international conference on applications of statistics and probability in civil engineering held in Vancouver, Canada
32. Li Q, Fan J, Nie J, Li Q, Chen Y (2010) Crowd-induced random vibration of footbridge and vibration control using multiple tuned mass dampers. J Sound Vib 329(19):4068-4092

33. Dimentberg M, Iourtchenko D, Bratus' A (2000) Optimal bounded control of steady-state random vibrations. Probab Eng Mech 15(4):381-386

34. Dimentberg MF, Bratus' AS (2000) Bounded parametric control of random vibrations. Proc R Soc Lond A Math Phys Eng Sci 456(2002):2351-2363

35. Roberts J, Spanos P (1986) Stochastic averaging: an approximate method of solving random vibration problems. Int J Non-Linear Mech 21(2):111-134

36. Soong T, Grigoriu M (1993) Random vibration of mechanical and structural systems. PTR Prentice-Hall Inc, New Jersey

37. Taflanidis AA, Scruggs JT, Beck JL (2008) Reliability-based performance objectives and probabilistic robustness in structural control applications. J Eng Mech 134(4):291-301

38. Grigoriu M (1995) Applied non-Gaussian processes : examples, theory, simulation, linear random vibration, and MATLAB solutions. Chap 2. Prentice-Hall, Englewood Cliffs, pp 5-40

39. Stathopoulos T (1980) PDF of wind pressures on low-rise buildings. J Struct Div ASCE 106:973-990

40. Ochi MK, Malakar SB, Wang W-C (1982) Statistical analysis of coastal waves observed during the Arsloe Project. UFL/COEL/TR045. University of Florida, Gainesville, FL, Coastal and Oceanographic Engineering Department

41. Radu A, Grigoriu M (2018) A Site-specific ground-motion simulation model: application for Vrancea earthquakes. Soil Dyn Earthq Eng 111:77-86

42. Pisal AY, Jangid RS (2016) Dynamic response of structures with tuned mass damper. Int J Adv Struct Eng 8(4):363-377

43. Chen MZQ, Papageorgiu C, Scheibe F, Wand FC, Smith MC (2009) The missing mechanical circuit element. IEEE circuits and systems magazine 9(1):10-26

44. Pachi A, Ji T (2005) Frequency and velocity of people walking. Struct Eng 83:36-40

45. Grigoriu M (2002) Stochastic calculus: applications in science and engineering. Birkhäuser, Boston

46. Singh P, Joshi SD, Patney RK, Saha K (2017) The fourier decomposition method for nonlinear and non-stationary time series analysis. Proc R Soc Lond A Math Phys Eng Sci 473(2199):1-27. https:// doi.org/10.1098/rspa.2016.0871

47. Cramér H, Leadbetter MR (2013) Stationary and related stochastic processes: sample function properties and their applications. Courier Corporation, New York

48. Crandall SH (1985) Non-Gaussian closure techniques for stationary random vibration. Int J Non-Linear Mech 20(1):1-8

49. Crandall SH, Chandiramani KL, Cook RG (1966) Some firstpassage problems in random vibration. J Appl Mech 33(3):532-538

50. Roberts JB (1976) First passage time for the envelope of a randomly excited linear oscillator. J Sound Vib 46(1):1-14

51. Caughey T, Stumpf H (1961) Transient response of a dynamic system under random excitation. J Appl Mech 28(4):563-566 